\title{
Research Analysis and Construction of Application of Deep Filtration Technology in Biopharmaceutical Process
}

\author{
Xin Zhou ${ }^{1}$ \\ ${ }^{1}$ Baoding Vocational and Technical College, Baoding City, Hebei Province 071051
}

\begin{abstract}
This article analyzes the difference between deep filtration technology and surface filtration technology. The author studied the specific application of deep filtration technology in clarification of fermentation broth, pyrogen removal filtration, small molecule purification, product purification and separation, and purification of macromolecules. This article analyzes the common problems during the application of deep filtration technology. The author puts forward suggestions to improve the resource sharing mechanism, standardize the management of filtering research institutions, increase research on new technologies, and do a good job of comprehensive training of personnel. The purpose of this article is to deepen people's understanding of deep filtration technology and promote the healthy economic development of the biopharmaceutical industry.
\end{abstract}

\section{Introduction}

At this stage, the biopharmaceutical process is advancing in the direction of rapid development, which also creates conditions for the treatment of many diseases. In the process of process application, deep filtration technology is a processing technology that is often used. Compared with surface filtration technology, this technology has better purification and separation effects and higher quality of finished products, which is also an important factor for the smooth development of this technology.

\section{The Difference Between Deep Filtration Technology and Surface Filtration Technology}

Deep filtration technology refers to the capture of impurities in the vertical depth. The depth filter media is generally composed of fibers. Compared with surface filtration, the interstitial channel of the deep filtration media has the characteristics of tortuous and slender, and the internal particles of the filtered medium are smaller than the interstitial channel. In this way, a better filtering effect can be achieved. The surface filtration is to capture particles through the surface of the filter material. In order to achieve the filtering function, we need to uniformly arrange the pore sizes of the upper and lower filter materials. There are many types of surface filter materials, such as filter screens and membranes. The advantages and disadvantages of the two filtering technologies are compared as follows. The two filtering technologies have different application advantages. In actual applications, we need to choose according to the actual situation to improve the reliability of the selection results.

Table1. Comparison of Deep Filtration Technology and Surface Filtration Technology

\begin{tabular}{|c|c|c|}
\hline Comparison Item & $\begin{array}{c}\text { Deep Filtration } \\
\text { Technology }\end{array}$ & $\begin{array}{c}\text { Surface Filtration } \\
\text { Technology }\end{array}$ \\
\hline Filter Media & $\begin{array}{c}\text { The membrane is a } \\
\text { disposable item }\end{array}$ & Can be reused \\
\hline Filter Content & Nominal Filter & Absolute Filtering \\
\hline $\begin{array}{c}\text { Pollution Holding } \\
\text { Capacity }\end{array}$ & Stronger & Weaker \\
\hline Service Life & Longer & Shorter \\
\hline $\begin{array}{c}\text { Material Handling } \\
\text { Method }\end{array}$ & $\begin{array}{c}\text { No Preprocessing } \\
\text { Required }\end{array}$ & $\begin{array}{c}\text { Need Pre- } \\
\text { processing }\end{array}$ \\
\hline
\end{tabular}

\section{Specific Application of Deep Filtration Technology in Biopharmaceutical Process}

\subsection{Clarified Fermentation Broth}

Under normal circumstances, the staff will carry out a rough separation operation for the remaining liquid after the biological fermentation work is completed. After this treatment, there will be many cell fragments, gel-packed substances, and intact cells (a small amount) in the fermentation broth. These substances will all exist in the supernatant, and these are impurities that will interfere with the final experimental results. In practical applications, the deep filtration technology can filter the active ingredients for multiple times, so as to achieve the purpose of exquisiteness. Based on previous practical 
experience, the staff will use mechanical interception and electrostatic adsorption to deal with it in the specific application process. The biggest advantage of this method in the application process is that the pressure drop in the fermentation broth can be controlled within a small range, thereby increasing the flux inside the structure. In this way, the efficiency of the deep filtration of the fermentation broth can be greatly improved, and the operation process in the application process will be simplified to a large extent. This also reduces the operating burden of the purification equipment and improves the rationality of the biopharmaceutical process.

\subsection{Pyrogen Filter}

In the context of the rapid development of medical engineering, the number of biomacromolecule drugs has increased sharply in recent years, but these macromolecular drugs have one of the biggest shortcomings that are thermal instability. Pyrogens are also called endotoxins, which are mostly attached to the outer wall of gram-negative bacteria, which is the socalled cell debris. This is a lipopolysaccharide substance, and the types of bacteria that produce endomycin are very different. As a result, the molecular weight of endomycin also ranges from tens of thousands to millions. Macromolecules with a molecular weight of one million will have an adverse effect on the body, and endomycin will cause great harm to the body. This is also an important reference indicator for evaluating the contamination of drugs by preparations. Using ZetaPlus series products to remove pyrogens can effectively solve this problem. Its principle is to use a machine with a molecular weight smaller than the pyrogen to intercept, and at the same time to use electrostatic adsorption to jointly remove the pyrogen. This will reduce labor intensity and increase product yield. Meanwhile, this also optimizes the quality of the product and saves costs.

\subsection{Small Molecule Refinement}

In biopharmaceuticals, there are macromolecular substances such as inner molds, and naturally small molecules such as antibiotics are also produced. Filtration, extraction, concentration, crystallization and other processes are important processes in the traditional production and processing of small molecule substances. But this process will lead to shortcomings such as too cumbersome production process, low yield, and high energy consumption. In the meantime, during the refining process, macromolecular impurities such as proteins, nucleic acids, and polysaccharides will also exist in trace amounts. These impurities will cause the medicine to be not pure enough, which will affect the quality of the medicine. In this process, if ZetaPlus depth filtration is used at the same time, macromolecular impurities can be effectively removed and the operation process can be simplified. In this way, invalid impurities with relatively high molecular weights such as starch, polysaccharides, proteins, and resins can be effectively removed. Compared with traditional chemical separation methods, this technology not only has high efficiency, simple operation, more operability, safety and reliability, but also has low cost and good economic benefits. It is being adopted more and more.

\subsection{Product Purification and Separation}

Biopharmaceutical technology involves many operations in the application process, and product purification and separation are also very important tasks. In this process, it will also use deep filtration technology to process it to obtain a purer sample. For example, many blood samples are used in biopharmaceutical processes. The blood sample contains a lot of contaminants, such as nucleic acids, viruses, and proteins. Hence, workers need to use the adsorption function of deep filtration technology to remove these impurities step by step before using it. In particular, some dangerous viruses or RNA fragments in blood samples can be cleaned up with this technology. In the process of technical treatment, it also has a strong dirt holding capacity. It can use the pressure drop environment provided by the technology to complete the smooth extraction of the sample. In the application process, the staff also need to pay attention to the temperature and operation process control work, so as to ensure that the sample can be separated smoothly as required, so as to obtain more reliable processing results.

\subsection{Refined Macromolecule}

Judging from the current development situation, macromolecular drugs have gradually become an important development trend of biopharmaceuticals. Compared with small molecular substances, macromolecular substances have poor thermal stability, and many factors need to be considered when preparing them. The smooth application of deep filtering technology can effectively improve the accuracy of the control results. In this process, it can not only clean up a large number of impurities in the sample, but also concentrate the active ingredients in it, thereby improving the reliability of the analysis results. The thermal denaturation method, if the heating process is not uniform, it will also destroy the integrity of the macromolecules. Another example is the high time cost and economic cost of the precipitation method in the use process. In summary, in the process of refining macromolecules, deep filtration technology has very high application value. Besides, the deep filtration program also has a very good application in the preparation of traditional Chinese medicine, which contains more macromolecule substances. The use of deep filtration technology can also be used to separate these high-molecular substances, so as to make medicines for different diseases to achieve the expected therapeutic goals. 


\section{Common Problems During the Application of Deep Filtration Technology}

\subsection{No Resource Sharing Mechanism has Been Established}

Judging from the current application situation, in the actual application of deep filtering technology, there is a problem that a resource sharing mechanism is not established. Its specific performance is as follows. Firstly, affected by the particularity of the industry, mastering new technologies means mastering a lot of wealth. Therefore, these technical resources will be transformed into a "technology monopoly" by some companies. According to data from 2013 to 2020 , more than $30 \%$ of biopharmaceutical technologies are currently monopolized, and technology sharing is low. This is also an important factor restricting the further development of technology. Secondly, there are many "useless efforts", that is, affected by the level of filtering technology mastery, many companies participating in market competition need to do a good job in improving their comprehensive capabilities under the background that they cannot obtain reliable shared resources. This also means that many resources will be wasted in the process of re-exploration, which also caused excessive waste of resources. Moreover, the emergence of this situation will also affect the quality of drugs circulating in the market, breed some unfair competition, and affect the sustainable development of the industry's economy [1].

\subsection{Unsound Filtering Research Institutions}

Judging from the current application situation, deep-layer filtering technology also has the problem of unsound filtering research institutions in practical applications. Its specific performance is as follows. Firstly, at this stage, there is no relatively complete filtration technology research institution in China, and the prepared research equipment, experimental environment, research samples and other items are not sufficient. The emergence of this kind of situation also greatly affects the further research of technology and reduces the practical value of the technology application process. Secondly, there are currently a large number of manufacturers dedicated to technology production, but the research strength is relatively weak. More than $60 \%$ of the manufacturers are "fighting on their own," and the scientific research team can only conduct research on problems. However, there is no opportunity for communication and learning, which will lead to long-term research failures when encountering R\&D bottlenecks. This will have a greater negative impact on the development of the entire industry and restrict the economic development speed of the entire industry [2].

\subsection{Difficulty in Process Control}

Based on the existing application experience, the deep filter technology also has the problem of greater difficulty in the process of filter research in practical applications. The specific manifestations are as follows: First, how to control the pressure difference in the precision filtration process when the sample is filtered. This will also be an important factor that directly affects the final filtering effect. However, the control of this content is difficult, and there are mechanical errors and accidental errors, which affect the smooth progress of control activities. Second, the content involved in the development of biopharmaceutical processes is numerous and complex. For example, the filtration process needs to filter many impurities, and the transient state of the material during filtration cannot be grasped in time. In many cases, the formation of biopharmaceuticals is formed in instantaneous changes, and the effects of finished products brought about by different changes are also quite different. For example, the active ingredients of morphine and the drug "opium" in medical drugs belong to isomers. However, the effects of different structures are very different [3].

\subsection{Inconsistent Comprehensive Literacy of Personnel}

Except to the above-mentioned application problems, in the current stage of development, deep filtration technology still has the problem of inconsistencies in the comprehensive literacy of personnel in practical applications. Its specific performance is as follows. Firstly, the talent pool is relatively small. The application of deep filtration technology to practice has a relatively short period of time. Compared with other industries, it is still relatively immature. This will also affect the focus of some companies on talent training, leading to inconsistencies in the comprehensive literacy of personnel in the research team, and affecting the continuation of scientific research activities. Secondly, there is relatively little cooperation among universities. At present, more than $70 \%$ of the external export of biopharmaceutical technology talents is based on the training of talents in corresponding majors in universities. However, there is less cooperation between enterprises and universities, and the number of counterpart talents that universities can cultivate is relatively small. Many students still need to study again after entering the enterprise, which also increases the cost of cultivating talents for the enterprise. Many companies are unwilling to accept these talents from colleges and universities, which not only makes many talents switch to other jobs, but also makes the market talent pool into a vicious circle [4].

\section{Suggestions on Improving the Application Effect of Deep Filtering Technology}

\subsection{Improve Resource Sharing Mechanism}

A sound resource sharing mechanism can not only avoid the problem of "technology monopoly", but also lay the foundation for the acceleration of the improvement of the 
technological system. In the specific practice process, first, the impact of the particularity of the industry requires the state to coordinate the economic interests of all parties and enhance the sharing of technological resources. This can reduce the "technology monopoly" and allow more technological research results to be shared among the society. In the initial stage, it can also do a good job in corporate cooperation and accomplish scientific resource sharing to a certain extent. This is also an important factor to promote the further development of technology. Second, increase the proportion of "useful work". That is, it is affected by the level of filtering technology mastery. In view of the inequality of shared resources, we need to do a good job of enterprise technology joint work. This can also reduce the resource consumption of enterprises in the process of re-exploration, apply more resources to the research and development process of new content, and improve the efficiency of resource utilization. In this way, in practical applications, the quality of drugs circulating in the market can be improved, and the breeding of unfair competition can be reduced, thereby promoting the sustainable development of the industry economy [5].

\subsection{Standardize the Management of Filtration Research Institutions}

Standardizing the management of filtration research institutions can create conditions for the in-depth research of deep filtration technology to speed up the speed of technological research. In practical applications, the following points should also be paid attention to. Firstly, the country needs to increase capital investment, establish a relatively complete filtration technology research institution, and improve the richness and completeness of research equipment, experimental environment, and research samples. This also creates conditions for the further research of technology and enhances the practical value of the technology application process. Secondly, in view of the large number of manufacturers engaged in technology production but the relatively weak scientific research capabilities, the government needs to play a pivotal role, find the balance between enterprises, and build mutual exchanges and cooperation platforms. Except to researching the problem, the research team can also enjoy the opportunity to communicate and learn. In this way, when encountering R\&D bottlenecks, the problem can be solved in a relatively short time by learning. This will have a very good beneficial impact on the development of the entire industry, thereby accelerating the economic development of the entire industry [6].

\subsection{Intensify Research on New Technologies}

Increasing the research on new technologies can make the control process more scientific and grasp more instantaneous change information. The staff need to do the following two points in the specific application process. First, the pressure difference in the precision filtration process needs to be controlled when the sample is filtered. Facing the influence of mechanical errors and accidental errors, it is necessary to focus on mechanical error control. At the same time, staff need to take into account accidental error monitoring, such as the use of information technology and dynamic monitoring technology to monitor the research and development process, to maximize the smooth progress of control activities. Second, considering that the biopharmaceutical process involves a wide range of content and complexity in the research and development process, it is also necessary to do a good job in the application of computer technology to sort out the instantaneous data of the material during the filtration. We can use this as a basis to explore the pharmaceutical technology, thereby speeding up the advancement of research activities [7].

\subsection{Do A Good Job in Comprehensive Training of Personnel}

Doing a good job of comprehensive personnel training can ensure the adequacy of the reserve of scientific research personnel, which also lays a reliable foundation for the smooth progress of follow-up activities. In specific practice, companies and schools also need to pay attention to the following points. First of all, companies should increase their talent reserves. With the continuous increase of practical experience in deep filtration technology, the focus of enterprises in talent training also needs to be inclined to the cultivation of such talents, and gradually improve the comprehensive quality of personnel in the research team. Secondly, strengthen university cooperation. The cooperation between enterprises and universities needs to continue to deepen. This can also ensure that universities can train more counterpart talents. Moreover, companies should provide some internship opportunities, so that students can directly devote themselves to research after entering the company, and reduce the cost of cultivating talents for the company [8].

\section{Conclusion}

In summary, improving the resource sharing mechanism can avoid the "technology monopoly" problem. Standardizing the management of filtration research institutions can create conditions for the in-depth research of deep filtration technology. Increasing the research of new technologies can make the control process more scientific. Doing a good job of comprehensive literacy training can ensure the adequacy of the reserve of scientific research personnel. The application of deep filtration technology to the research of biopharmaceutical processes has positive significance for the rapid economic development of the industry.

\section{References}

1. Liu Jing. Discussion on the practice of deep filtration technology in biopharmaceutical process [J]. Family medicine. Medical treatment and drug selection, 2019(02): 185-186.

2. Peng Anjie, Liu Xiaolan. Research on the application 
of deep filtration technology in biopharmaceutical processes[J]. Science and Technology Innovation, 2019(01): 164-165.

3. Kang Wei. The application of deep filtration technology in biopharmaceutical process[J]. China Food, 2018(22):147.

4. Zhang Dongliang. The practice of deep filtration technology in biopharmaceutical process[J]. Construction Science and Technology, 2017(23): 133134.

5. Xiao Ye. Application of deep filtration technology in biopharmaceutical process[J]. Information Recording Materials, 2017, 18(02): 83-84.

6. Ma Chunyu. Analysis of the application of deep filtration technology in biopharmaceutical technology[J]. Heilongjiang Science and Technology Information, 2017(01):55.

7. He Qing. The application of deep filtration technology in biopharmaceutical process[J]. Electronic Journal of Clinical Medicine Literature, 2016, 3(04): 632-633.

8. Du Yunlai. My humble opinion on the application of deep filtration technology in biopharmaceutical technology[J]. Modern Health, 2016(16): 58-60. 